\title{
Density-functional theory for classical fluids and solids
}

\author{
C. Ebner \\ Department of Physics, Ohio State University, Columbus, Ohio 43210 \\ H. R. Krishnamurthy and Rahul Pandit \\ Department of Physics, Indian Institute of Science, Bangalore 560012, India
}

\begin{abstract}
We formulate a density-functional theory that is capable of describing simultaneously the solid, liquid, and gas phases of a simple classical material. The formalism can be reduced to the EbnerSaam-Stroud theory for the liquid-gas case and to a generalized version of the RamakrishnanYoussouff theory for the liquid-solid case. The theory requires as input the direct correlation functions of a uniform fluid. As an example we apply the formalism to the calculation of the phase diagram of a system with Lennard-Jones intermolecular interactions. We obtain the correlation functions from a closure scheme proposed by Zerah and Hansen [J. Chem. Phys. 84, 2336 (1986)]. The calculated density-temperature phase diagram compares favorably with those obtained from numerical simulations of the same model system. We also compute the equations of state in the solid and fluid phases.
\end{abstract}

\section{INTRODUCTION}

There have been many studies of the condensation of a gas into a liquid and the freezing of a liquid into a solid within the framework of density-functional theory. ${ }^{1}$ The freezing transition in particular has presented a serious test of the formalisms. An early formulation, by Ramakrishnan and Youssouff, 2,3 and one on which much later work has been based, ${ }^{1,4,5}$ expands the liquid-solid freeenergy difference to second order in powers of the density difference $\rho_{s}(\mathbf{r})-\rho_{l} ; \rho_{s}$ is the density of the solid and $\rho_{l}$ is that of the coexisting liquid. This theory gives reasonable results for a model hard-sphere system but fails to produce crystallization of systems of particles interacting with softer cores. ${ }^{6} 8$ This failure can be overcome by keeping higher-order terms in the expansion. ${ }^{6,7}$ For the particular case of a Lennard-Jones (LJ) potential, the theory produces crystallization, but the liquid and solid densities and the pressure at the phase transition are rather high, ${ }^{9}$ a shortcoming which, again, is partially remedied by including some third-order terms in the expansion." However, the inclusion of higher-order terms when treating hard spheres actually gives rise to inferior results in comparison with those from the secondorder expansion. ${ }^{12}$ Hence there are basic questions as to the accuracy and predictive power of the functionals employed in each of these calculations.

A somewhat different approach to constructing a density functional, pioneered by Tarazona ${ }^{13}$ and others, ${ }^{14-20}$ is to express the solid's free energy as that of a fluid at some "effective" density. Various of these theories have produced quite good results in certain applications. For example, Curtin and Ashcroft ${ }^{21}$ obtained a quantitatively correct phase diagram, in comparison with Monte Carlo simulations, for a LJ system using the weighted-density approximation $^{14}$ (WDA) to calculate the properties of a hard-sphere system and then doing a perturbation expansion around this system. And de Kuijper et al. ${ }^{9}$ had similar success for the freezing of a LJ system using the modified weighted-density approximation ${ }^{20}$ (MWDA), provided they treated the attractive part of the interaction via mean-field theory. However, when they applied the MWDA to the full LJ potential, there was no freezing transition at all at temperatures below about four times the critical temperature. See Ref. 9 for a more complete description of these and other interesting results from attempts to apply various density functionals to the freezing of simple systems.

In this paper we develop a density-functional formalism which has the useful qualities that it is relatively easy to motivate and simple to apply, with no input other than the two-particle direct correlation functions of the uniform fluid. Moreover, it can be used in situations where all three phases - gas, solid, and liquid - of a simple system are present and has the appealing property that for the gas-liquid subsystem it can be reduced to the densityfunctional theory of Ebner, Saam, and Stroud, ${ }^{22}$ while for the liquid-solid subsystem it reduces to a generalized version of the density-functional theory of Ramakrishnan and Yussouff. ${ }^{2}$ Finally, we apply the functional to the calculation of the phase diagram and thermodynamics of a LJ system. The results are qualitatively very good; quantitatively speaking, they are not fully satisfactory as regards the liquid-solid transition, although they are generally superior to those obtained from other functionals that do not make recourse to perturbation or mean-field treatments of the attractive part of the interaction. ${ }^{9}$

In Sec. II we develop our density-functional theory. 
We also review in this section the scheme of Zerah and Hansen $^{23}$ which we employ for obtaining the direct correlation functions of a LJ fluid. Section III describes our calculations and results for the correlation functions, thermodynamic functions of the fluid and solid, and phase diagram. Finally, in Sec. IV we summarize and conclude with a brief comparison of our results with those of other density-functional theories.

\section{DENSITY-FUNCTIONAL THEORY AND CLOSURE SCHEME}

\section{A. Density-functional theory}

We begin by expanding the grand free-energy functional for a nonuniform system in a functional Taylor series about a reference density $\rho_{0}$,

$$
\begin{aligned}
-\beta W \equiv \beta\left(\Omega-\Omega_{\mathrm{id}}\right)= & -\beta W_{0}-C^{(1)}\left(\rho_{0}\right) \int d^{3} r_{1}\left[\rho\left(\mathbf{r}_{1}\right)-\rho_{0}\right]-\frac{1}{2 !} \int d^{3} r_{1} d^{3} r_{2} C^{(2)}\left(\mathbf{r}_{1}-\mathbf{r}_{2} ; \rho_{0}\right)\left[\rho\left(\mathbf{r}_{1}\right)-\rho_{0}\right]\left[\rho\left(\mathbf{r}_{2}\right)-\rho_{0}\right] \\
& -\frac{1}{3 !} \int d^{3} r_{1} d^{3} r_{2} d^{3} r_{3} C^{(3)}\left(\mathbf{r}_{1}, \mathbf{r}_{2}, \mathbf{r}_{3} ; \rho_{0}\right)\left[\rho\left(\mathbf{r}_{1}\right)-\rho_{0}\right]\left[\rho\left(\mathbf{r}_{2}\right)-\rho_{0}\right]\left[\rho\left(\mathbf{r}_{3}\right)-\rho_{0}\right]-\cdots,
\end{aligned}
$$

where $\Omega_{\mathrm{id}}$ is the free energy of the ideal gas, $\beta$ is the inverse temperature, and the $C^{(n)}$ are the $n$-particle direct correlation functions. Also,

$$
\begin{aligned}
\beta \Omega_{\mathrm{id}}= & \int d^{3} r \rho(\mathbf{r}) \ln \left[\frac{\mathrm{p}(\mathrm{r})}{\rho_{\mathrm{id}}}\right] \\
& -\beta \mu \int d^{3} r \rho(\mathbf{r})-\int d^{3} r \rho(\mathbf{r}),
\end{aligned}
$$

where $\mu$ is the chemical potential and $\rho_{\mathrm{id}}=\lambda^{-3}, \lambda$ being the thermal wavelength.

Now, since the free energy must be independent of $\rho_{0}$, we have the condition that

$$
\begin{gathered}
\partial(\beta W) \\
\partial \rho_{0}
\end{gathered}=0
$$

for any $\rho_{0}$. Consequently we find

$$
\begin{aligned}
& \frac{\partial}{\partial \rho_{0}}\left(\beta W_{0}\right)=C^{(1)}\left(\rho_{0}\right), \\
& \frac{\partial}{\partial \rho_{0}}\left[C^{(1)}\left(\rho_{0}\right)\right]=\int d^{3} r_{2} C^{(2)}\left(\mathbf{r}_{1}-\mathbf{r}_{2} ; \rho_{0}\right), \\
& \bar{\partial}_{\rho_{0}}\left[C^{(2)}\left(\mathbf{r}-\mathbf{r}^{\prime} ; \rho_{0}\right)\right]=\hat{\mathbf{J}} d^{3} r_{1} C^{(3)}\left(\mathbf{r}, \mathbf{r}^{\prime}, \mathbf{r}_{1} ; \rho_{0}\right),
\end{aligned}
$$

and so on.

Consider first a constant density system, $p(r)=p$. We can compute the integrals in Eq. (1) in terms of zerowave-vector Fourier components of the correlation functions

$$
\begin{aligned}
-\frac{\beta W}{V} \equiv & -\beta \omega \\
= & -\beta \omega_{0}-C^{(1)}\left(\rho_{0}\right)\left(\rho-\rho_{0}\right)-\frac{1}{2} \widetilde{C}_{0}^{(2)}\left(\rho-\rho_{0}\right)^{2} \\
& -\frac{1}{3 !} \widetilde{C}_{00}^{(3)}\left(\rho-\rho_{0}\right)^{3}-\cdots .
\end{aligned}
$$

More generally, we define the Fourier transforms of the correlation functions by

$$
\begin{gathered}
\tilde{C}_{\mathbf{q}}^{(2)}=\int d^{3} r C^{(2)}(\mathbf{r}) e^{-i \mathbf{q} \cdot \mathbf{r}}, \\
\widetilde{C}_{\mathbf{q}_{1}, \mathbf{q}_{2}}^{(3)}=\int d^{3} r_{1} d^{3} r_{2} C^{(3)}\left(\mathbf{r}_{1}-\mathbf{r}_{3}, \mathbf{r}_{2}-\mathbf{r}_{3}\right) \\
X e^{-i\left[\mathbf{q}_{1} \cdot\left(\mathbf{r}_{1}-\mathbf{r}_{3}\right)+\mathbf{q}_{2} \cdot\left(\mathbf{r}_{2}-\mathbf{r}_{3}\right)\right]},
\end{gathered}
$$

and so on. Continuing from Eq. (5), we can formally sum the expansion if we make use of the Fourier transforms of Eqs. (4), which are

$$
\begin{aligned}
& \frac{\partial \widetilde{C}_{\mathbf{q}}^{(2)}\left(\rho_{0}\right)}{\partial \rho_{0}}=\widetilde{C}_{\mathbf{q},-\mathbf{q}}^{(3)}\left(\rho_{0}\right), \\
& \frac{\partial \widetilde{C}_{\mathbf{q}_{1}, \mathbf{q}_{2}}^{(3)}\left(\rho_{0}\right)}{\partial \rho_{0}}=\widetilde{C}_{\mathbf{q}_{1}, \mathbf{q}_{2},-\mathbf{q}_{1}-\mathbf{q}_{2}}^{(4)}\left(\rho_{0}\right),
\end{aligned}
$$

etc., to obtain

$$
\begin{aligned}
& -\beta \omega=-\beta \omega_{0}-C^{(1)}\left(\rho_{0}\right)\left(\rho-\rho_{0}\right)-\sum_{n=0}^{\infty} \widetilde{C}_{0, \ldots, 0}^{(n+2:>} \frac{\left(\rho-\rho_{0}\right)^{1+2}}{(n+2) !} \\
& =-\beta \omega_{0}-C^{(1)}\left(\rho_{0}\right)\left(\rho-\rho_{0}\right) \\
& -\sum_{n=0}^{\infty} \frac{\left(\rho-\rho_{0}\right)^{n+2}}{(n+2) !} \frac{\partial^{n} \tilde{C}_{0}^{(2)}\left(\rho_{0}\right)}{\partial \rho_{0}^{n}} \text {. }
\end{aligned}
$$

The summation can be done exactly in the form of integrals of the correlation function $\widetilde{C}_{0}^{(2)}(\rho)$ over the density, leading to

$-\beta \omega=-\beta \omega_{0}-C^{(1)}\left(\rho_{0}\right)\left(\rho-\rho_{0}\right)-\int_{\rho_{0}}^{\rho} d \rho_{1} \int_{\rho_{0}}^{\rho_{1}} d \rho_{2} \widetilde{C}_{0}^{(2)}\left(\rho_{2}\right)$,

and the order of integrations can be exchanged to obtain

$$
\begin{aligned}
-\beta \omega= & -\beta \omega_{0}-C^{(1)}\left(\rho_{0}\right)\left(\rho-\rho_{0}\right)-\int_{\mu} \int_{0}^{\rho} d \rho_{2}\left(\rho-\rho_{2}\right) \widetilde{C}_{0}^{(2)}\left(\rho_{2}\right) \\
= & -\beta \omega_{0}-C^{(1)}\left(\rho_{0}\right)\left(\rho-\rho_{0}\right) \\
& -\int_{\rho_{0}}^{\rho} d \rho_{1}\left[C^{(1)}\left(\rho_{1}\right)-C^{(1)}\left(\rho_{0}\right)\right],
\end{aligned}
$$

where we have made use of the fact that $\partial C^{(1)}(\rho) / \partial \rho=\widetilde{C}_{0}^{(2)}$; see the second of Eqs. (4). Finally, Eq. (10) can be simplified to read

$$
-\beta \omega=-\beta \omega_{0}-\int_{P O}^{\rho} d \rho_{1} C^{(1)}\left(\rho_{1}\right) .
$$

Notice that the condition for equilibrium, $\partial \omega / \partial \rho=(3$, implies that the equilibrium density is given by

$$
\rho=\rho_{\mathrm{id}} e^{\beta \mu+C^{(1)}(\rho)} .
$$


Now let us choose $\rho_{0}=0$. Then $\omega_{0}=0$ and $C^{(1)}\left(\rho_{0}\right)=0$. Hence

$$
\begin{aligned}
-\beta \omega & =-\int_{0}^{\rho} d \rho_{1} C^{(1)}\left(\rho_{1}\right) \\
& =-\int_{0}^{\rho} d \rho_{2}\left(\rho-\rho_{2}\right) \widetilde{C}_{0}^{(2)}\left(\rho_{2}\right)
\end{aligned}
$$

is our final result for the free energy of a uniform system.

Consider next the case of a uniform solid. Start once again from Eqs. (1) and (2). Set $\rho_{0}=0$ as before and expand the density in a Fourier series,

$$
\rho(\mathbf{r})=\sum_{\mathbf{G}} \rho_{\mathrm{G}} e^{i \mathbf{G} \cdot \mathbf{r}} .
$$

where the G's are reciprocal-lattice vectors. Then the free energy, Eq. (1), may be written as

$$
\begin{aligned}
\frac{\beta \Omega}{V}= & \frac{1}{v} \int_{v} d^{3} r \rho(\mathbf{r}) \ln \left(\frac{\mathrm{p}(\mathrm{r})}{\mathrm{Pid}}\right)-\rho_{s}-\beta \mu \rho_{s} \\
- & \left(\frac{1}{2 !} \sum_{\mathrm{G}} \widetilde{C}_{\mathrm{G}}^{(2)}(0) \rho_{\mathrm{G}} \rho-\mathrm{G}\right. \\
& \left.+\frac{1}{3 !} \sum_{\mathbf{G}_{1}, \mathrm{G}_{2}} \widetilde{C}_{\mathbf{G}_{1}, \mathrm{G}_{2}}^{(3)}(0) \rho_{\mathrm{G}_{1}} \rho_{\mathrm{G}_{2}} \rho_{-\mathrm{G}_{1}-\mathrm{G}_{2}}+\cdots\right)
\end{aligned}
$$

where $v$ is the volume of a primitive cell of the crystal and $\rho_{s}$ is $\rho_{\mathrm{G}=0}$, the mean density in the solid.

We next collect terms with all $\mathbf{G}_{i}$ equal to zero, two $\mathbf{G}_{i}$ not equal to zero, three $\mathbf{G}_{i}$ not equal to zero, etc., and use relations (7) to get

$$
\begin{aligned}
\frac{\beta \Omega}{V}= & \frac{1}{v} \int_{v} d^{3} r \rho(\mathbf{r}) \ln \left(\frac{\mathrm{p}(\mathrm{r})}{\operatorname{Pid}}\right]-\rho_{s}-\beta \mu \rho_{s} \\
& -\int_{0}^{\rho_{s}} d \rho^{\prime}\left(\rho_{s}-\rho^{\prime}\right) \widetilde{C}_{s}^{(2)}\left(\rho^{\prime}\right)-\frac{1}{2} \sum_{\mathbf{G} \neq 0} \widetilde{C}_{\mathrm{G}}^{(2)}\left(\rho_{s}\right) \rho_{\mathbf{G}} \rho_{-\mathbf{G}} \\
& -\frac{1}{3 !} \sum_{\mathbf{G}_{1} \neq 0, \mathbf{G}_{2} \neq 0, \mathbf{G}_{1}+\mathbf{G}_{2} \neq 0} \widetilde{\boldsymbol{C}}_{\mathbf{G}_{1}, \mathbf{G}_{2}}^{(3)}\left(\rho_{s}\right) \rho_{\mathbf{G}_{1}} \rho_{\mathbf{G}_{2}} \rho_{-\mathbf{G}_{1}-\mathbf{G}_{2}}
\end{aligned}
$$

In the application that follows, we drop terms involving $C^{(n)}$ with $n=3$ or larger and so have a simple prescription for the grand free energy of the solid in terms of the direct two-particle correlation function of a uniform system with the average density of the solid. Also, in practice we work with the Helmholtz free energy which can be obtained by adding a term $\beta \mu \rho_{s}$ to the right-hand side of Eq. (16). Finally, we set the ideal gas reference density to unity. Hence we have the following expression for the Helmholtz free-energy density, in units of $\beta^{-1}$, of a solid with density $\rho_{s}$ :

$$
\begin{aligned}
\frac{\beta F_{s}}{V}= & \frac{1}{v} \int_{v} d^{3} r\left[\mathrm{p}(\mathrm{r}) \ln \left[\frac{\mathrm{p}(\mathrm{r})}{\operatorname{Pid}}\right]-\rho_{s}\right] \\
& -\int_{0}^{\rho_{s}} d \rho^{\prime}\left(\rho_{s}-\rho^{\prime}\right) \widetilde{C}_{0}\left(\rho^{\prime}\right)-\frac{1}{2} \sum_{\mathrm{G} \neq 0} \widetilde{C}_{\mathrm{G}}\left(\rho_{s}\right) \rho_{\mathrm{G}} \rho_{-\mathrm{G}} \cdot
\end{aligned}
$$

The superscript (2) has been dropped from the symbol for the direct correlation function.

Notice that, if we have a uniform system so that $\rho_{\mathrm{G}}=0$ for $\mathbf{G} \neq 0$, Eq. (17) reduces to an appropriate expression for the Helmholtz free-energy density of that uniform system, as may be seen by making the appropriate modification of Eq. (13). It is in fact identical to the expression for the free energy of a uniform fluid given by Ebner, Saam, and Stroud. ${ }^{22}$ Further, Eq. (17) is essentially the same as the free-energy functional of Ramakrishnan and Youssouff, except that the uniform reference density about which it is expanded is the solid density and not the liquid density which is equivalent to summing exactly all terms in the free energy which are precisely of second order in $\rho_{\mathbf{G}}$ with $\mathbf{G} \neq \mathbf{0}$ and summing terms of all orders in the uniform density change.

Implementation of Eq. (17) involves, first, obtaining $C^{(2)}$ for uniform systems and, second, minimization of the free-energy functional with respect to $\rho(\mathbf{r})$ at some fixed value of $\rho_{s}$. We have obtained the correlation functions by methods described in Sec. II B. As for the minimization, there are two standard procedures. One is to expand the density as a Fourier series, Eq. (14), and to minimize with respect to the amplitudes $\rho_{\mathbf{G}}$ for $\mathbf{G} \neq 0$. The second is to parametrize the density as a periodic sum of Gaussian functions and to minimize with respect to appropriate parameters.

We have chosen the second route. The density is written as

$$
\rho(\mathbf{r})=\rho_{s} v\left[\frac{\gamma}{2 \pi}\right]^{3 / 2} \sum_{\mathbf{R}} e^{-\gamma(\mathbf{r}-\mathbf{R})^{2} / 2},
$$

where the vectors $\mathrm{R}$ are lattice translations of the assumed crystalline structure ( $\mathrm{fcc}$ ). For given $\rho_{s}$, we find the values of the nearest-neighbor distance or lattice constant $a$ and of the parameter $\gamma$ from the condition that the free energy be minimized. In this way one obtains a curve of free energy as a function of $a$. Notice that the primitive cell volume $v$ depends on $a$ and that in the absence of vacancies, the product $\rho_{s} v \equiv b$ would be unity as there would be one particle in each primitive cell of volume $v$. Typically, the minimum is obtained for a value of $a$ at which $b$ is a few percent smaller than one (see Sec. III).

The evaluation of the ideal-gas part of the free energy involves only an integration over the logarithm of the density. Because the overlap of the Gaussian functions on neighboring sites is extremely small for all cases of interest (see Sec. III), it can be neglected and the integral done analytically, 


$$
\begin{aligned}
\frac{1}{v} \int_{v} d^{3} r \rho(\mathbf{r}) \ln [\rho(\mathbf{r})] & \approx \frac{1}{v} \int_{v} d^{3} r b\left[\frac{\gamma}{2 \pi}\right]^{3 / 2} e^{-\gamma r^{2} / 2}\left\{\ln \left[b\left[\frac{y}{2 \pi}\right]^{3 / 2}\right]-\frac{\gamma}{2} r^{2}\right\} \\
& =\frac{b}{V}\left\{\ln \left[b\left[\frac{\gamma}{2 \pi}\right]^{3 / 2}\right]-\frac{3}{2}\right\} .
\end{aligned}
$$

As for the term involving $\mathrm{C}$, it is evaluated as follows. First,

$$
\begin{aligned}
\sum_{\mathrm{G} \neq 0} \tilde{C}_{\mathrm{G}} \rho_{\mathrm{G}} \rho_{-\mathrm{G}}= & \sum_{\mathrm{G} \neq 0} \frac{1}{V^{2}} \int_{V} d^{3} r d^{3} r^{\prime} \rho(\mathbf{r}) \rho\left(\mathbf{r}^{\prime}\right) e^{-i \mathbf{G} \cdot\left(\mathbf{r}-\mathbf{r}^{\prime}\right)} \widetilde{C}_{\mathrm{G}} \\
= & \frac{1}{V} \int_{V} d^{3} r d^{3} r^{\prime} \rho(\mathbf{r}) \rho\left(\mathbf{r}^{\prime}\right) C\left(\mathbf{r}-\mathbf{r}^{\prime} ; \rho_{s}\right) \\
& -\mathrm{C}_{v}\left(p_{-}\right) \rho_{s}^{2} .
\end{aligned}
$$

Integration is over the (ultimately infinite) volume $V$ of the system. Expanding the density as a sum of Gauss- ians, Eq. (18), we can show that

$$
\begin{aligned}
& \frac{1}{V} \int d^{3} r d^{3} r^{\prime} C\left(\mathbf{r}-\mathbf{r}^{\prime} ; \rho_{s}\right) \rho(\mathbf{r}) \rho\left(\mathbf{r}^{\prime}\right) \\
& \quad=\frac{1}{v} b^{2}\left[\frac{\gamma}{4 \pi}\right]^{3 / 2} \sum_{\mathbf{R}} / \ell^{l^{3} r} C\left(r ; \rho_{s}\right) e^{-\gamma(\mathbf{r}+\mathbf{R})^{\prime} / 4} .
\end{aligned}
$$

Next, we integrate over the directions of $r$ and express the result as a sum over shells of atoms in the lattice structure. After this is done and the pieces of the free energy assembled, we have

$$
\begin{aligned}
& \frac{\beta F_{s}}{V}=-\frac{v}{v}\left\{\ln \left[b\left[\frac{\gamma}{2 \pi}\right]^{3 / 2}\right]-\frac{3}{2}\right\}-{ }_{\mathrm{ft}} \int_{0}{ }^{\sim} \rho_{\tilde{d}} \rho\left(\rho \quad \mathrm{P}^{\prime} s\right) \widetilde{C}_{0}(\rho)+\frac{\rho_{s}^{2}}{2} \widetilde{C}_{0}\left(\rho_{s}\right)-\frac{b^{2} \gamma^{3 / 2}}{4 \sqrt{\pi} v} \int_{0}^{\infty} d r r^{2} C\left(r ; \rho_{s}\right) e^{-\gamma r^{2} / 4} \\
& -\frac{b^{2} \sqrt{\gamma}}{4 \sqrt{\pi} v} \sum_{s \neq 0} \frac{N_{s}}{R_{s}} \int_{-\infty}^{\infty} d r\left(R_{s}+r\right) C\left(R_{s}+r ; \rho_{s}\right) e^{-\gamma r^{2} / 4} \text {. }
\end{aligned}
$$

$R_{s}$ and $N_{s}$ are, respectively, the radius of the 5th shell of atoms and the number of atoms in this shell. From this point, the integration is completed numerically and the free energy minimized with respect to $b$ and $\gamma$.

The free energy of the solid must be compared with that of a uniform fluid to obtain the equilibrium phase diagram of the system. The corresponding expression for the free energy of a fluid with density $\rho_{f}$ is

$$
\frac{\beta F_{f}}{V} \equiv \rho_{f} \ln \left(\rho_{f}\right)-\rho_{f}-\int_{{ }_{v}}{ }^{\lambda} d \rho\left(\rho-\rho_{f}\right) \widetilde{C}_{0}(\rho) .
$$

\section{B. Closure scheme of Zerah and Hansen}

Extending the work of Rogers and Young, ${ }^{24}$ Zerah and Hansen $^{23}$ have proposed a closure scheme which yields the pair distribution function of a simple classical fluid as the solution of an integral equation. The essence of this closure scheme is to interpolate smoothly in real space between the hypernetted-chain (HNC) closure, which is known to work well for the long-ranged part of a potential such as the van der Waals potential, and the soft-core mean-spherical approximation (SMSA), which is known to work well for potentials with a soft repulsive part and a small attractive part.

The procedure is as follows. First, the interparticle potential $v(r)$ is divided into two pieces, $v=v_{1}+v_{2}$ with

$$
v_{1}(r)= \begin{cases}v(r)-v_{m}, & r<r_{m} \\ 0, & r>r_{m}\end{cases}
$$

and

$$
v_{2}(r)= \begin{cases}v_{m}, & r<r_{m} \\ v(r), & r>r_{m}\end{cases}
$$

where $v_{m}$ is the minimum value of the potential, located at $r=r_{m}$. Next, the proposed closure relation is

$g(r)=e^{-\beta v_{1}(r)}\left[1+\frac{e^{a(r)\left[h(r)-C(r)-\beta v_{2}(r)\right]}-1}{a(r)}\right]$,

where $g(r)$ is the pair distribution function and $h(r)=g(r)-1$ is the total correlation function. The "switching function" $a(r)$ goes to zero at small $r$ and to unity at large $r$ so that the closure reduces to, respectively, the SMSA closure and the HNC closure in these limits. Following Zerah and Hansen (who in turn follow Rogers and Young), we shall take the switching function to be of the form

$$
a(r)=1-e^{-\alpha r}
$$

with the single parameter a determined by the condition that the virial and compressibility equations of state are identical.

Combining Eq. (26) with the Ornstein-Zernike relation,

$$
h(r)=C(r)+\rho \int d^{3} r^{\prime} h\left(\mathbf{r}-\mathbf{r}^{\prime}\right) C\left(\mathbf{r}^{\prime}\right),
$$

and using the Lennard-Jones (6-12) potential, 


$$
v(r)=4 \epsilon\left[\left(\frac{\sigma}{r}\right]^{12}-\left[\frac{\sigma}{r}\right)^{6}\right],
$$

we have obtained, except in the region of spinodal decomposition, fluid pair distribution functions and direct correlation functions for temperatures and densities of importance for the purposes of calculating the phase diagram. In the region of unstable fluid, we have obtained the direct correlation functions by simultaneously interpolating in density between correlation functions in the gas and liquid regions and extrapolating down in temperature from correlation functions at temperatures above the spinodal line. The procedure is the one described and used in Ref. 22.

\section{CALCULATIONS AND RESULTS}

\section{A. Correlation functions}

Using Eqs. (26) and (28) with the intermolecular potential (29), we solve for the $C(r)$ and $g(r)$ at a given density and temperature on a grid of evenly spaced points in $r$ using an iterative procedure. The spacing of the points is $0.03 \sigma$ and the functions are determined out to $r=10.5 \sigma$ The iterations are done in one of two ways; the first is to use as the input for a given iteration a mix of the input and output of the previous iteration, and the second, which has been described in detail by $\mathrm{Ng}^{25}$ is to construct an optimized input for a given iteration by making use of the input and output from several (typically two to four) previous iterations. The latter method is more complicated but usually produces much faster convergence. The criterion for convergence is that, in the final iteration, the input and output for $C(\boldsymbol{r})$ should differ nowhere by more than $10^{-6}$.

The correlation functions have been found for reduced densities $p^{*} \equiv \rho \sigma^{3}$ up to 1.2 and for reduced temperatures $T^{*} \equiv k T / \epsilon$ between 0.5 and 1.5. Intervals of $T^{*}$ and $p^{*}$ are usually 0.1 and 0.05 , respectively, but smaller intervals are employed in some instances. In the region of spinodal decomposition (within the closure scheme employed) we obtain direct correlation functions from the interpolation and extrapolation procedure described in Ref. 22.

Zerah and Hansen ${ }^{23}$ have discussed the correlation functions produced by the closure scheme Eq. (26) and have made detailed comparisons with results of simulations for the same system as that considered here; consequently we shall restrict our discussion to a few representative results. Figure 1 displays the reduced switching parameter $\alpha^{*} \equiv \alpha \sigma$ as a function of $p^{*}$ (except in the region of spinodal decomposition) at several temperatures; it is relatively small at large densities and becomes large at small densities where the HNC closure, which is obtained in the limit $\alpha_{0} \rightarrow \infty$, is more accurate. In Fig. 2 we show the structure factor

$$
S(k)=1+\rho \int d^{3} r[g(r)-1] e^{i \mathbf{k} \cdot \mathbf{r}},
$$

at several densities and temperatures close to points of two- or three-phase coexistence and also close to the criti-

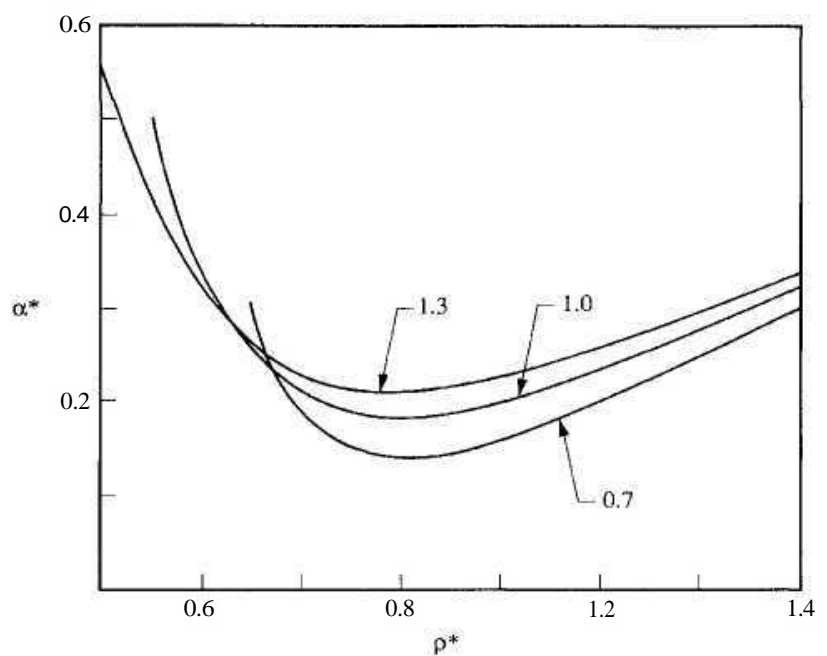

FIG. 1. The reduced switching parameter $a^{*}$ is plotted againstp* at temperatures $T^{*}=0.7,1.0$, and 1.3.

cal point. These figures demonstrate the competition of the peaks at zero wave number and at wave numbers characterizing the inverse lattice spacing of the solid.

\section{B. Thermodynamics and phase diagram}

We have evaluated the Helmholtz free-energy density $/=F / V$, chemical potential $\mu$, and pressure $P$ in the fluid and solid phases as functions of $p^{*}$ and $T^{*}$. In the following, the reduced thermodynamic functions $f^{*} \equiv \sigma^{3} f / \epsilon, \mu^{*} \equiv \mu / \epsilon$, and $P^{*} \equiv \sigma^{3} P / \epsilon$ are presented. In the fluid phases the free energy is easily evaluated, given the direct correlation functions, from Eq. (23); in the

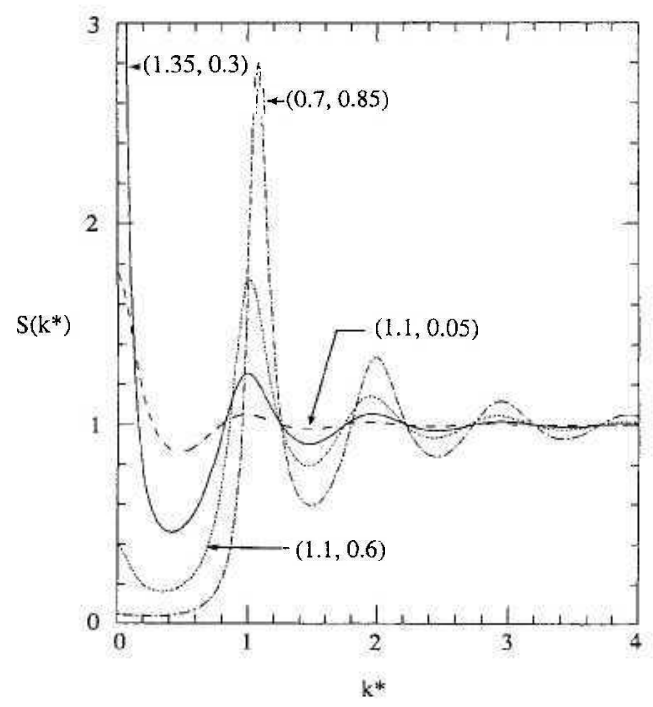

FIG. 2. The structure factor is shown as a function of $k^{*}=k \sigma / 2 \pi$ for $T^{*}=1.35, \mathrm{p}^{*}=0.30 \quad(-) ; T^{*}=1.10$, $\mathrm{p}^{*}=0.05 \quad\left(-\mathrm{r}^{*}=1.10, \mathrm{p}^{*}=0.60 \quad(-)\right.$ and $T^{*}=0.70, \mathrm{p}^{*}=0.85(-)$. Each curve is labeled with the corresponding $\left(T^{*}, \rho^{*}\right)$. 
solid phase we find /* from Eq. (22) by numerically evaluating the integrals using Gaussian quadrature and interpolation of the tabulated correlation functions. It is necessary to do this many times while minimizing the free energy with respect to $b$ and $\gamma$ for given $p$ and $T$. One hundred shells of lattice sites are retained in the sum. Figure 3 displays $\gamma^{*}=\gamma \sigma^{2}$ for the equilibrium solid as a function of $\mathrm{p}^{*}$ at several temperatures while Fig. 4 is the analogous plot of $b$.

Once $f^{*}$ is known, for either type of phase, the chemical potential is found from $\mu^{*}=\partial f^{*} / \partial \rho^{*}$, and then the pressure (or the grand free energy) follows from $P^{*}=\mu^{*} \rho^{*}-f^{*}$. Phase equilibria are determined from knowledge of $f^{*}\left(\rho^{*}\right)$ for the appropriate phases by making (numerically) a double-tangent construction. Figure 5 shows $P^{*}, \mu^{*}$, and $f^{*}$ for the fluid phases as functions of $p^{*}$ at temperatures of (a) 0.7, (b) 1.0, and (c) 1.3. Figure 6 shows the same for the solid phase. From the curves for $l^{*}$ in Fig. 5 one can make the appropriate double-tangent construction to obtain the gas-liquid coexistence densities; Fig. 7 is a representative double-tangent construction for liquid-solid coexistence and is at $T^{*}=1.0$. Finally, the density-temperature phase diagram is presented in Fig. 8; Fig. 9 displays more detailed plots of the temperature dependences of the liquid density, the solid density, and the pressure as functions of the temperature at coexistence. Included for comparison are results from Monte Carlo simulations ${ }^{26-28}$ of the LJ system.

Several remarks are in order. First, the gas-liquid coexistence curve as determined here is in considerably better agreement with both Monte Carlo simulations of the LJ system and with real fluids which the LJ system approximates, such as argon, than the one found in Ref. 22. Given that the same functional was used to find the fluid free energy in both calculations, the improvement must be attributed to the closure scheme employed in determining the correlation functions. In Ref. 22, the Percus-Yevick (PY) closure was employed; in the present work, the closure Eq. (26) proposed by Zerah and Han$\operatorname{sen}^{23}$ has been invoked. The implication is clearly that the latter is significantly superior. Moreover, the entire phase diagram compares favorably with results from simulations of the LJ system, cf. Figs. 8 and 9, especially given the relatively simple and straightforward density functional that we employ and the absence of adjustable parameters. For example, we find a triple-point temperature of $T^{*}=0.665$; the experimental value for $\operatorname{argon}^{29}$ is close to 0.70 . It is worth noting in this context that we also computed the properties of the solid phase and the solid-liquid phase boundary employing PY correlation functions. The results for the latter were considerably less satisfactory than those presented here; for example, the triple-point temperature was much larger, about 1.1 in reduced units.

\section{SUMMARY}

We have constructed a relatively simple densityfunctional formalism for classical systems which allows for solid, liquid, and gas phases. It extends and consolidates the theories of Ebner, Saam, and Stroud ${ }^{22}$ for fluid

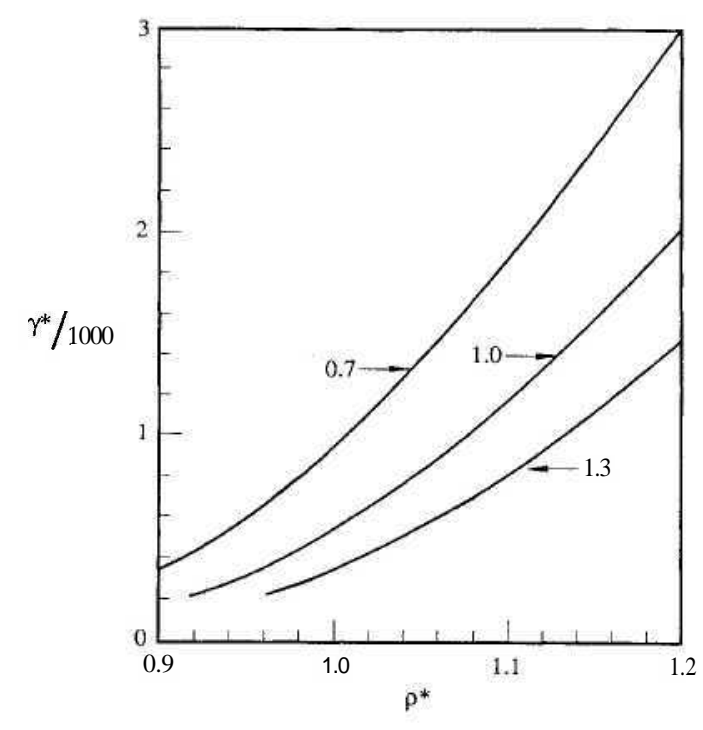

FIG. 3. The parameter $\gamma^{*}$ for the equilibrium solid is shown as a function $o f p^{*}$ for $T^{*}=0.7,1.0$, and 1.3.

systems and of Ramakrishnan and Youssouff ${ }^{2}$ for solid systems. The sole input needed for application of the theory is direct correlation functions of the uniform fluid; there are no fitting parameters. In the application of the formalism presented here, we have obtained these correlation functions using a closure scheme proposed by Zerah and Hansen ${ }^{23}$ which is demonstrably superior to other simple approximate closures such as the PY and HNC approximations.

We have applied the formalism to the calculation of the phase diagram of a Lennard-Jones system and have also calculated some thermodynamic properties-free energy, pressure, and chemical potential-in the fluid

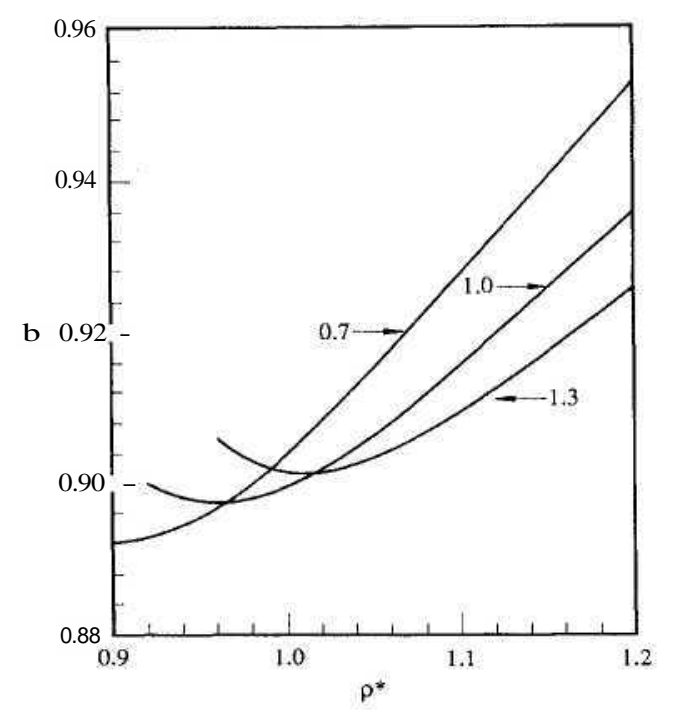

FIG. 4. The parameter $b$ for the equilibrium solid is shown as a function of $\mathrm{p}^{*}$ for $\mathrm{r}^{*}=0.7,1.0$, and 1.3 . 
TABLE I. Comparison of freezing parameters at $T^{*}-1$ obtained from the Haymet and Oxtoby (HO) formulation as implemented in Ref. 9, the MWDA + MF of Ref. 9, our calculations (present), and Monte Carlo (MC) simulations, Refs. 26-28. The quantities presented are the reduced densities $\rho_{l}^{*}$ and $\rho_{s}^{*}$ of the liquid and solid at coexistence, the relative density change $\mathrm{Sp} / \mathrm{p}$; upon freezing, the reduced pressure $P^{*}$ at freezing, and the Lindemann parameter $L$. This table is adapted from Table 2 of Ref. 9. We note that MC simulations were not reported at this temperature in Refs. 26-28; the entries in the table are obtained by interpolating between results at higher and lower temperatures.

\begin{tabular}{clcccc}
\hline \hline Theory & Pf & $\rho_{s}^{*}$ & $\mathrm{Sp} / \mathrm{p}$, & $P^{*}$ & $L$ \\
\hline HO & 0.974 & 1.094 & 0.12 & 6.0 & 0.105 \\
MWDA+MF & 0.88 & 1.025 & 0.16 & 3.2 & 0.100 \\
Present & 0.944 & 1.084 & 0.15 & 4.8 & 0.12 \\
MC & 0.910 & 1.005 & 0.10 & 3.6 & 0.14 \\
\hline
\end{tabular}
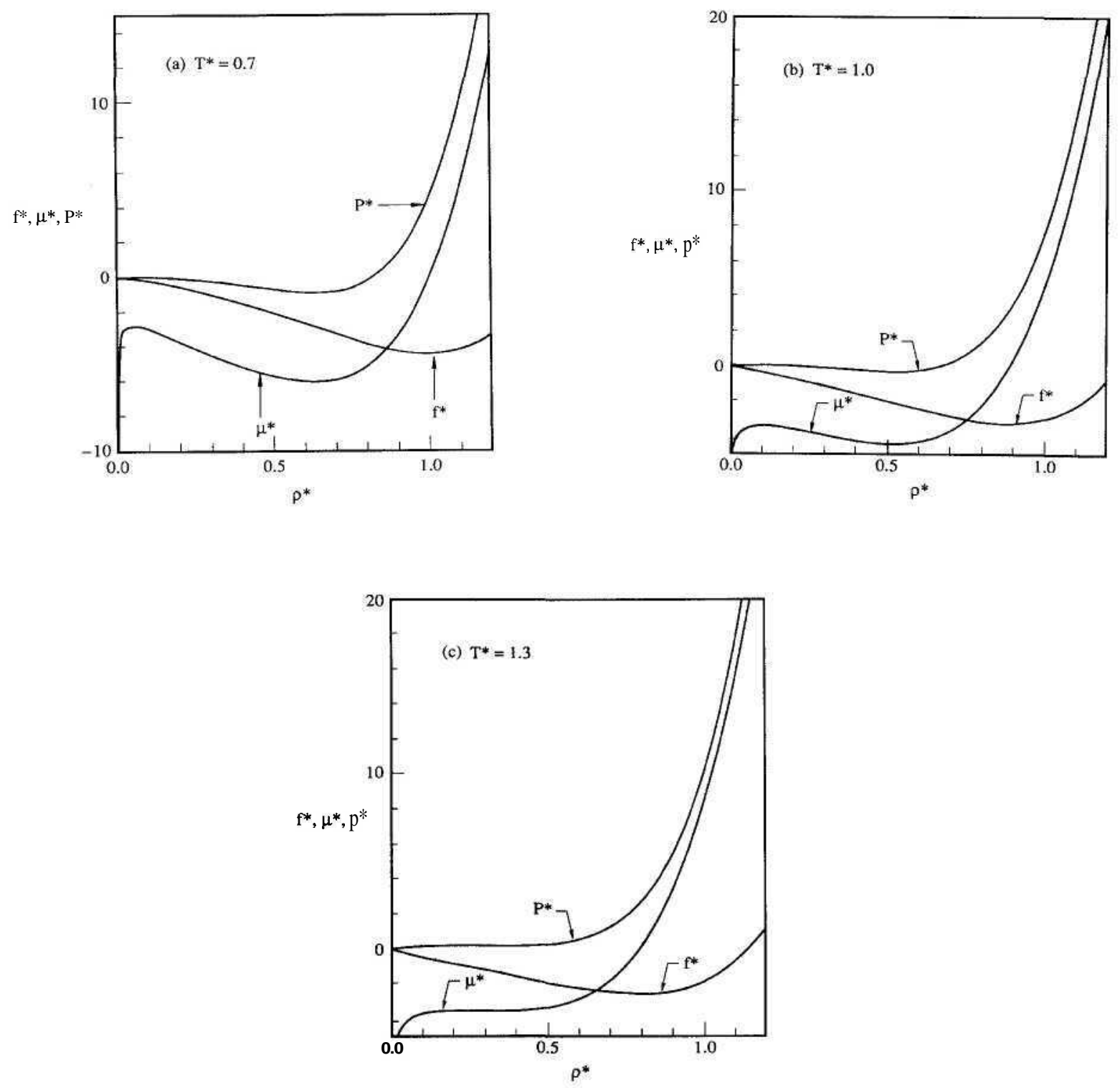

FIG. 5. The thermodynamic functions $P^{*}, /^{*}$, and $\mu^{*}$ in the fluid phases are shown as functions $o f p^{*}$ at temperatures $T^{*}$ of (a) 0.7 , (b) 1.0, and (c) 1.3 . 

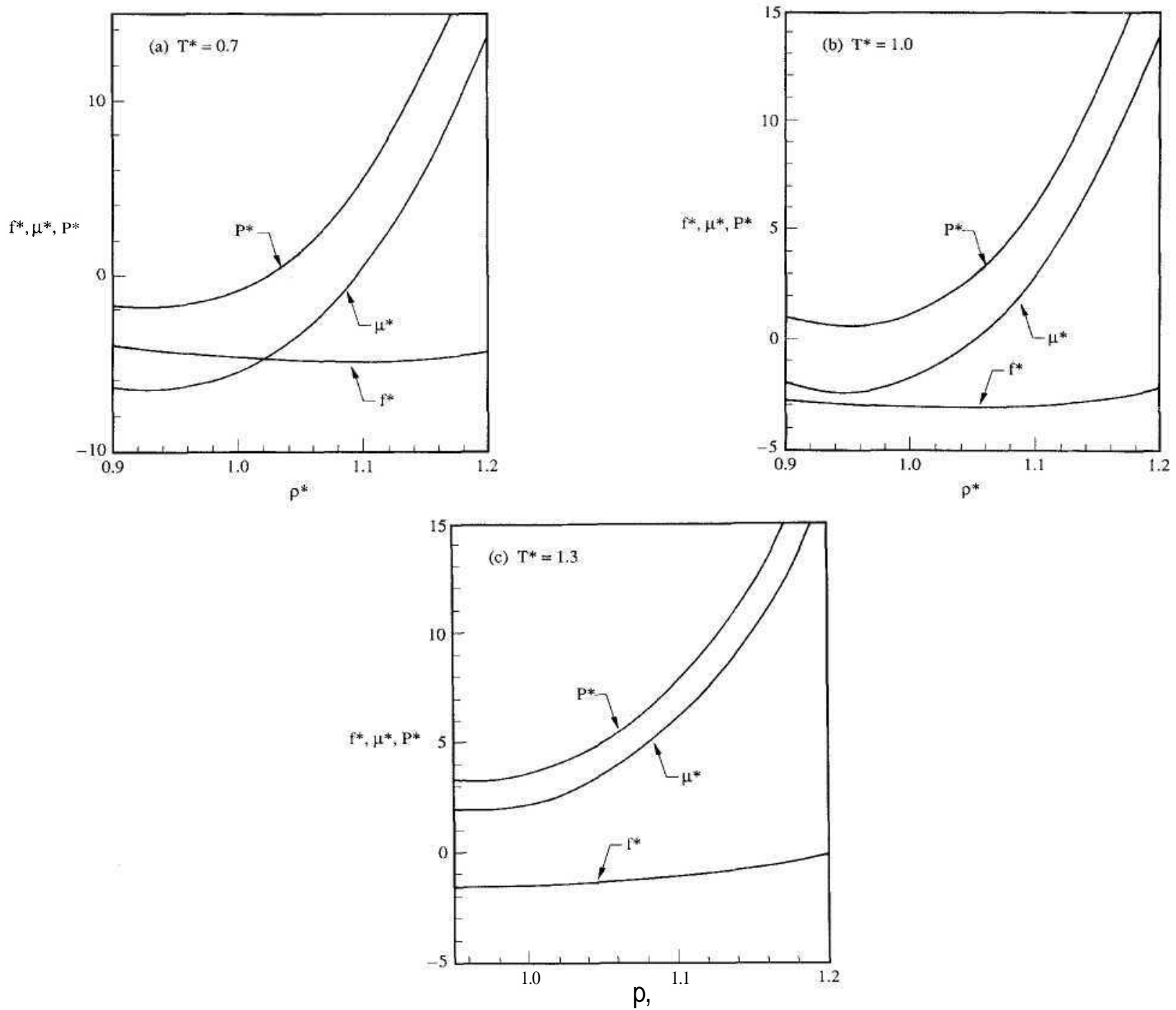

FIG. 6. The thermodynamic functions $P^{*}, /^{*}$, and $\mu^{*}$ in the solid phase are shown as functions of $\mathrm{p}^{*}$ at temperatures $T^{*}$ of (a) 0.7 , (b) 1.0 , and (c) 1.3 .

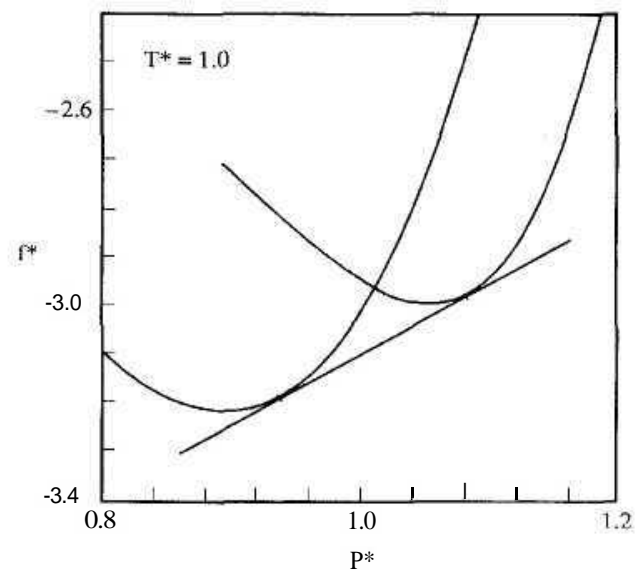

FIG. 7. The common-tangent construction for the liquid and solid phases at $T^{*}=1.0$; the curves are the reduced Helmholtz free energies of the liquid and solid phases.

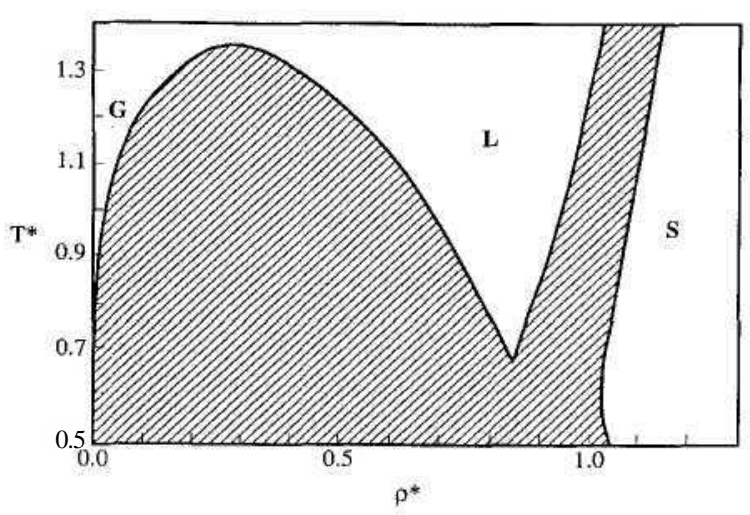

FIG. 8. The calculated density-temperature phase diagram of the Lennard-Jones system, showing single-phase solid (5), liquid $(L)$, and gaseous $(G)$ regions and two-phase regions (shaded). 


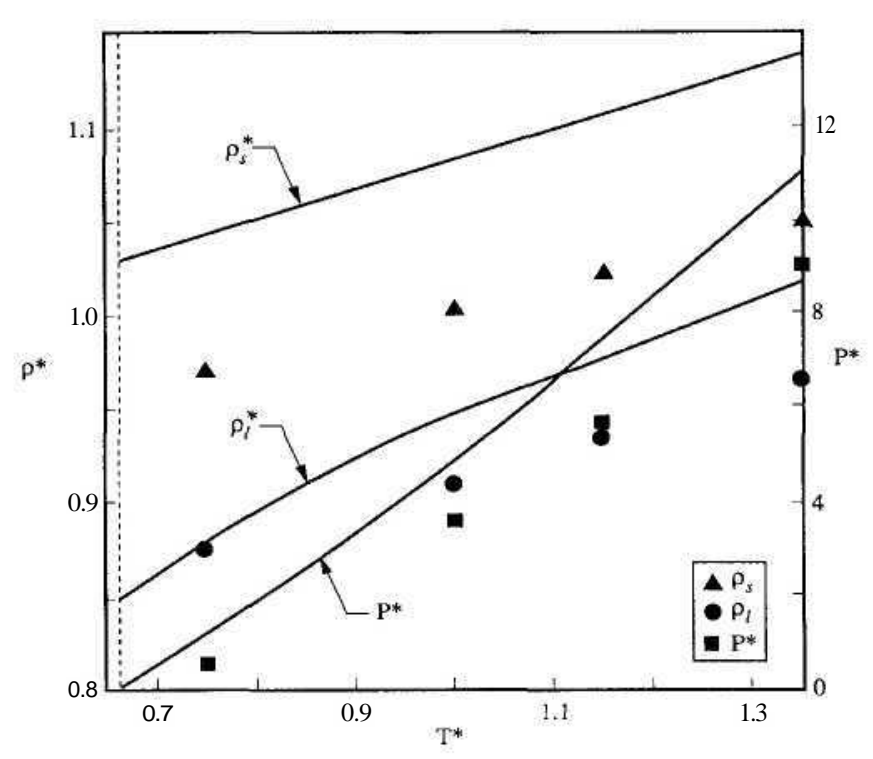

FIG. 9. The reduced pressure $P^{*}$, liquid density $\rho_{i}^{*}$, and solid density $p f$ at phase coexistence are shown as functions of $T^{*}$; results for these quantities from Monte Carlo simulations, Refs. 26-28, are shown as $\bullet$, and A, respectively.

and solid phases. The phase diagram in particular is in good agreement with experiments on appropriate real systems, such as argon, ${ }^{29}$ and with Monte Carlo simulations of the Lennard-Jones system. In Table I we compare freezing parameters determined here with those from Ref. 4 and from Monte Carlo simulations; ${ }^{26-28}$ also included are results from the MWDA + MF scheme of de Kuijper et al. ${ }^{9}$

To the best of our knowledge, the only other densityfunctional theory that obtains gas, liquid, and solid phases and phase boundaries in good agreement with Monte Carlo simulations is the weighted-density approximation (WDA) of Curtin and Ashcroft. ${ }^{21}$ (An earlier version of this theory is due to Tarazona. ${ }^{13}$ ) Our densityfunctional theory is computationally less taxing than the WDA; however, it is not straightforward to compare our theory with the Curtin-Ashcroft implementation of the WDA for the Lennard-Jones system for the following reasons: (1) Curtin and Ashcroft obtain their expression for the free energy of an inhomogeneous liquid by expanding about a reference hard-sphere system which is treated by using the WDA; it is not clear whether the WDA would fail for the Lennard-Jones system without this expansion as a modified version of it (see below) does.
(2) Curtin and Ashcroft use an approximation for determining the direct correlation function which we believe is not as accurate as the Zerah-Hansen ${ }^{23}$ closure which we employ. (3) Lastly they parametrize the Lennard-Jones potential as a sum of two exponentials. Denton and Ashcroft $^{20}$ have recently proposed a modified weighteddensity approximation (MWDA) that combines the advantages of the WDA with the computational simplicity of the original Ramakrishnan-Yussouff ${ }^{2}$ densityfunctional theory. However, as de Kuijper et al. have shown, the MWDA does not yield a solid phase at all, either stable or metastable, at temperatures $T^{*}<5$. In order to obtain a solid phase they treat the attractive forces in a mean-field fashion, leading to the MWDA + MF scheme, cf. Ref. 9. Further, the modified effective liquid approximation (MELA) of Baus ${ }^{19}$ also does not yield a solid phase in the range of $1<T^{*}<10$, as pointed out in Ref. 9. Thus we believe that our density-functional theory is superior, both qualitatively and quantitatively, to most other DF theories and that it is comparable to the best, e.g., the WDA of Curtin and Ashcroft or the MWDA + MF of de Kuijper et al. Also, it is at the same time computationally as simple as the original EbnerSaam-Stroud ${ }^{22}$ and Ramakrishnan-Yussouff ${ }^{2}$ theories.

In the future we hope to apply the formalism to other, more complex problems involving nonuniform systems containing gas, liquid, and solid phases of a simple material; a case in point is the surface-melting ${ }^{30}$ phenomenon. To study this and similar problems, it will be necessary to build appropriate nonuniformities into the parametrization of the density. Preliminary investigations of the surface melting problem in particular indicate that this added complication is well within the capabilities of available computing machinery.

\section{ACKNOWLEDGMENTS}

We wish to thank T. V. Ramakrishnan and S. Sengupta for useful discussions. This work was supported in part by the University Grants Commission and Department of Science and Technology (India) and by National Science Foundation Grant Nos. DMR-8705606 and DMR9014679. Two of us (R.P. and H.R.K.) thank the Ohio State University Department of Physics for hospitality while part of this work was being done. The numerical work was done using the Ohio State University Department of Physics VAX 8650 and the Ohio Supercomputer Center CRAY Y-MP8/864.
${ }^{1}$ Density-functional theories of simple classical systems are reviewed by R. Evans, Adv. Phys. 28, 143 (1979); A. D. J. Haymet, Prog. Solid State Chem. 17, 1 (1986); Ann. Rev. Phys. Chem. 38, 89 (1987); M. Baus, J. Stat. Phys. 48, 1129 (1987).

${ }^{2}$ T. V. Ramakrishnan and M. Youssouff, Solid State Commun. 21, 389 (1977).

${ }^{3}$ T. V. Ramakrishnan and M. Youssouff, Phys. Rev. B 19, 2775 (1979).
${ }^{4}$ A. D. J. Haymet and D. J. Oxtoby, J. Chem. Phys. 74, 2559 (1981).

${ }^{5}$ D. J. Oxtoby and A. D. J. Haymet, J. Chem. Phys. 76, 6262 (1982).

${ }^{6}$ J.-L. Barrat, Europhys. Lett. 3, 523 (1987).

7J.-L. Barrat, J.-P. Hansen, G. Pastore, and E. M. Waisman, J. Chem. Phys. 86, 6360 (1987).

${ }^{8}$ M. Rovere and M. P. Tosi, J. Phys. C 18, 3445 (1985). 
${ }^{9}$ A. de Kuijper, W. L. Vos, J.-L. Barrat, J.-P. Hansen, and J. A. Schouten(unpublished).

${ }^{10}$ C. Marshall, B. B. Laird, and A. J. D. Haymet, Chem. Phys. Lett. 122, 320 (1985).

${ }^{11}$ B. B. Laird, J. D. McCoy, and A. D. J. Haymet, J. Chem. Phys. 87, 5449 (1987).

${ }^{12}$ W. A. Curtin, J. Chem. Phys. 88, 7050 (1988).

${ }^{13}$ P. Tarazona, Mol. Phys. 52, 81 (1984); Phys. Rev. A 31, 2672 (1985).

${ }^{14}$ W. A. Curtin and N. W. Ashcroft, Phys. Rev. A 32, 2909 (1985).

${ }^{15}$ M. Baus and J. L. Colot, Mol. Phys. 55, 653 (1985).

${ }^{16}$ F. Iglói and J. Hafner, J. Phys. Chem. 19, 5799 (1986).

${ }^{17}$ T. F. Meister and D. M. Kroll, Phys. Rev. A 31, 4055 (1985).

${ }^{18}$ R. D. Groot and J. P. van der Eerden, Phys. Rev. A 36, 4356 (1987).

${ }^{19}$ M. Baus, J. Phys.: Cond. Matter 1, 3131 (1989).

${ }^{20}$ A. R. Denton and N. W. Ashcroft, Phys. Rev. A 39, 4701
(1989).

${ }^{21}$ W. A. Curtin and N. W. Ashcroft, Phys. Rev. Lett. 56, 2775 (1986).

${ }^{22}$ C. Ebner, W. F. Saam, and D. G. Stroud, Phys. Rev. A 14, 2264 (1976).

${ }^{23}$ G. Zerah and J.-P. Hansen, J. Chem. Phys. 84, 2336 (1986).

${ }^{24}$ F. J. Rogers and D. A. Young, Phys. Rev. A 30, 999 (1984).

${ }^{25}$ K.-C. Ng, J. Chem. Phys. 61, 2680 (1974).

${ }^{26}$ J.-P. Hansen and L. Verlet, Phys. Rev. 184, 151 (1969).

${ }^{27}$ J.-P. Hansen, Phys. Rev. A 2, 221 (1970).

${ }^{28}$ J.-P. Hansen and I. R. McDonald, Theory of Simple Liquids (Academic, New York, 1976).

${ }^{29}$ See, e.g., Ref. 28 , for summaries of many measured properties of argon and other rare gases.

${ }^{30}$ See, e.g., A. Trayanov and E. Tosatti, Phys. Rev. Lett. 59, 2207 (1987); Phys. Rev. B 38, 6961 (1988), and references therein. 